\title{
Estimation of Sobolev-type embedding constant on domains with minimally smooth boundary using extension operator
}

\author{
Kazuaki Tanaka ${ }^{1 *}$, Kouta Sekine ${ }^{2}$, Makoto Mizuguchi ${ }^{1}$ and Shin'ichi Oishi ${ }^{2,3}$
}

\author{
"Correspondence: \\ imahazimari@fuji.waseda.jp \\ ${ }^{1}$ Graduate School of Fundamental \\ Science and Engineering, Waseda \\ University, 3-4-1 Okubo, \\ Shinjuku-ku, Tokyo, 169-8555, Japan \\ Full list of author information is \\ available at the end of the article
}

\begin{abstract}
In this paper, we propose a method for estimating the Sobolev-type embedding constant from $W^{1, q}(\Omega)$ to $L^{p}(\Omega)$ on a domain $\Omega \subset \mathbb{R}^{n}(n=2,3, \ldots)$ with minimally smooth boundary (also known as a Lipschitz domain), where $p \in(n /(n-1), \infty)$ and $q=n p /(n+p)$. We estimate the embedding constant by constructing an extension operator from $W^{1,9}(\Omega)$ to $W^{1,9}\left(\mathbb{R}^{n}\right)$ and computing its operator norm. We also present some examples of estimating the embedding constant for certain domains.
\end{abstract}

MSC: 46E35

Keywords: embedding constant; extension operator; Sobolev inequality

\section{Introduction}

Let $\Omega \subset \mathbb{R}^{n}(n=2,3, \ldots)$ be a domain with minimally smooth boundary (also known as a Lipschitz domain), the definition of which will be introduced in Definition 2.6. We are concerned with a concrete value of a constant $C_{p}(\Omega)$ for the embedding $W^{1, q}(\Omega) \hookrightarrow L^{p}(\Omega)$, i.e., $C_{p}(\Omega)$ satisfies

$$
\|u\|_{L^{p}(\Omega)} \leq C_{p}(\Omega)\|u\|_{W^{1, q}(\Omega)}, \quad \forall u \in W^{1, q}(\Omega)
$$

where $p \in(n /(n-1), \infty), q=n p /(n+p)$, and the norm $\|\cdot\|_{W^{1, q}(\Omega)}$ denotes the $\sigma$-weighted $W^{1, q}$ norm defined as

$$
\|\cdot\|_{W^{1, q(\Omega)}}^{q}:=\|\nabla \cdot\|_{L^{q}(\Omega)}^{q}+\sigma\|\cdot\|_{L^{q}(\Omega)}^{q}
$$

for given $\sigma>0$.

Since the Sobolev-type embedding theorems are important in studies on partial differential equations (PDEs), many studies have investigated such theorems and their applications, e.g., [1-16]. In particular, a concrete value of the embedding constant is indispensable for verified numerical computation and computer-assisted proof for PDEs; see, e.g., $[9-11,13]$. The best constant in the classical Sobolev inequality on $\mathbb{R}^{n}$ was independently shown by Aubin [1] and Talenti [14] in 1976 (see Theorem B.1). Moreover, since all elements $u$ in $W_{0}^{k, q}(\Omega)$, the commonly defined closure of $C_{0}^{\infty}(\Omega)$, can be regarded as elements

(c) 2015 Tanaka et al. This article is distributed under the terms of the Creative Commons Attribution 4.0 International License (http://creativecommons.org/licenses/by/4.0/), which permits unrestricted use, distribution, and reproduction in any medium, provided you give appropriate credit to the original author(s) and the source, provide a link to the Creative Commons license, and indicate if changes were made. 
of $W^{k, q}\left(\mathbb{R}^{n}\right)$ by zero extension outside $\Omega$, a constant for the embedding $W_{0}^{1, q}(\Omega) \hookrightarrow L^{p}(\Omega)$ can be estimated for a general domain $\Omega \subset \mathbb{R}^{n}$ by calculating the best constant of the classical embedding constant. Although, as a limited result, one can find in [17] a formula that gives a concrete value of a constant for the embedding $W^{1,2}(\Omega) \hookrightarrow L^{p}(\Omega)$ on a square $\Omega \subset \mathbb{R}^{2}$, little is known about a concrete value of a constant for the embedding $W^{1, q}(\Omega) \hookrightarrow L^{p}(\Omega)$ on general domains.

To estimate a concrete value of the embedding constant defined by (1), we construct a linear and bounded operator $E$ from $W^{1, q}(\Omega)$ to $W^{1, q}\left(\mathbb{R}^{n}\right)$ such that $(E u)(x)=u(x)$ for all $x \in \Omega$, which is called an extension operator from $W^{1, q}(\Omega)$ to $W^{1, q}\left(\mathbb{R}^{n}\right)$. We then estimate bounds for the operator norm $A_{q}(\Omega)$ of $E$ satisfying

$$
\|\nabla(E u)\|_{L^{q\left(\mathbb{R}^{n}\right)}} \leq A_{q}(\Omega)\left(\|\nabla u\|_{L^{q}(\Omega)}+\sigma^{1 / q}\|u\|_{L^{q}(\Omega)}\right), \quad \forall u \in W^{1, q}(\Omega),
$$

which leads to bounds for the embedding constant. Several construction methods for extension operators have been proposed. For example, a summary of the reflection method, which was originally proposed by Whitney [16] and Hestenes [7], can be found in, e.g., [18, 19]. In addition, Calderón [5] constructed a $k$-dependent extension operator from $W^{k, p}(\Omega)$ to $W^{k, p}\left(\mathbb{R}^{n}\right), 1<p<\infty$, on domains satisfying the uniform cone condition. Stein [12] subsequently showed that a $k$-independent extension operator from $W^{k, p}(\Omega)$ to $W^{k, p}\left(\mathbb{R}^{n}\right)$, $1 \leq p \leq \infty$, can be constructed on domains with minimally smooth boundary. The extension theorems of Calderón and Stein are summarized in, e.g., [18]. Furthermore, Stein's extension theorem was recently generalized by Rogers [20] to the so-called $(\varepsilon, \delta)$-domain, the notation of which was originally introduced in [21]. Although many studies have investigated such extension operators (see, e.g., [22-25]), little is known about the concrete values of their operator norms.

The main contribution of this paper is to propose a formula that gives a concrete value of $A_{q}(\Omega)$ for the extension operator constructed by Stein's method. Stein first constructed an extension operator on the special Lipschitz domain and then expanded it to that on domains with minimally smooth boundary. In his method, the regularized distance plays an important role; it is a $C^{\infty}$ function that approximates the distance from a given closed set $S \subset \mathbb{R}^{n}$ to any point in its complement $S^{c}$. Subsequent to the development of Stein's construction method, the regularized distance was generalized to a one-parameter family of smooth functions by Fraenkel [26]. In this paper, we construct an extension operator using Stein's method with the generalized regularized distance to derive the embedding constant.

\section{Preparation}

Throughout this paper, the following notation is used:

- $\mathbb{N}=\{1,2,3, \ldots\}$ and $\mathbb{N}_{0}=\{0,1,2, \ldots\}$;

- $B(x, r)$ is an open ball with center $x$ and radius $r \geq 0$;

- for any point $x=\left(x_{1}, x_{2}, \ldots, x_{n}\right) \in \mathbb{R}^{n}$ and any $p>0$, define $|x|_{p}:=\left(\left|x_{1}\right|^{p}+\left|x_{2}\right|^{p}+\cdots+\left|x_{n}\right|^{p}\right)^{\frac{1}{p}}$

- if no confusion arises, we denote $|\cdot|=|\cdot|_{2}$;

- for any set $S \subset \mathbb{R}^{n}, S^{c}$ is its complementary set, and $\bar{S}$ is its closure set;

- for any set $S \subset \mathbb{R}^{n}$ and any $\varepsilon>0$, define $S^{\varepsilon}:=\left\{x \in \mathbb{R}^{n}: B(x, \varepsilon) \subset S\right\}$;

- for any point $x \in \mathbb{R}^{n}$ and any set $S \subset \mathbb{R}^{n}$, define $\operatorname{dist}(x, S):=\inf \{|x-y|: y \in S\}$; 
- for any function $f, \operatorname{supp} f$ denotes the support of $f$;

- for any function $f$ over $\mathbb{R}, f^{\prime}$ denotes the ordinary derivative of $f$;

- for any function $f$ over $\mathbb{R}^{n}(n=2,3, \ldots), \partial_{x_{i}} f$ denotes the partial derivative of $f$ with respect to the $i$ th component $x_{i}$ of $x$.

Let $L^{p}(\Omega)(1 \leq p<\infty)$ be the functional space of $p$ th-power Lebesgue-integrable functions over $\Omega$. Let $W^{k, p}(\Omega)(k \in \mathbb{N}, 1 \leq p<\infty)$ be the $k$ th-order $L^{p}$ Sobolev space on $\Omega$; in particular, we denote $H^{k}(\Omega):=W^{k, 2}(\Omega)$.

Definition 2.1 (Mollifier) A nonnegative function $\rho \in C^{\infty}\left(\mathbb{R}^{n}\right)$ is said to be a mollifier if

$$
\rho(x)=0 \quad \text { for }|x| \geq 1 \quad \text { and } \quad \int_{\mathbb{R}^{n}} \rho(x) d x=1 .
$$

For example, the function

$$
\rho(x):= \begin{cases}c \exp \left(\frac{-1}{1-|x|^{2}}\right), & |x|<1, \\ 0, & |x| \geq 1\end{cases}
$$

becomes a mollifier, where $c$ is chosen such that $\int_{\mathbb{R}^{n}} \rho(x) d x=1$.

In the following lemma, the existence of a $C^{\infty}$ function approximating Lipschitz continuous functions is guaranteed.

Lemma 2.2 (Fraenkel [26]) Let $f: \mathbb{R}^{n} \rightarrow \mathbb{R}$ be a function satisfying the Lipschitz continuous condition, i.e., for some $M>0$,

$$
|f(x)-f(y)| \leq M|x-y|, \quad \forall x, y \in \mathbb{R}^{n} .
$$

Suppose that there is an open set $G \subset \mathbb{R}^{n}$ such that $f(x)>0$ for all $x \in G$. Then, for any given $\varepsilon \in(0,1)$, there is a function $g \in C^{\infty}(G)$ such that, for all $x \in G$,

$$
(1+\varepsilon)^{-2} f(x) \leq g(x) \leq(1-\varepsilon)^{-2} f(x)
$$

and

$$
\left|\frac{\partial^{\alpha}}{\partial x^{\alpha}} g(x)\right| \leq P_{\alpha} M^{\alpha}\{\varepsilon f(x)\}^{1-|\alpha|}, \quad \forall \alpha \in \mathbb{N}_{0}^{n} \text { with }|\alpha| \geq 1 .
$$

Here, $P_{\alpha}$ is a constant depending only on $\alpha$.

We can find in the proof of Lemma 2.2 (see [26]) that one of the concrete values of $P_{\alpha}$ can be derived as follows.

Lemma 2.3 Let $\rho$ be the mollifier defined in (4). Let $\rho_{*}: \mathbb{R} \rightarrow \mathbb{R}$ be a function such that $\rho_{*}(|x|)=\rho(x), x \in \mathbb{R}^{n}$. The multiindex $\alpha$ is written as $\alpha=\beta+\gamma$ for $\beta, \gamma \in \mathbb{N}_{0}^{n}$ with $|\gamma|=1$. Then, inequality (6) holds for

$$
P_{\alpha}=\int_{\mathbb{R}^{n}} \frac{\left|\frac{\partial^{\beta}}{\partial x^{\beta}} \rho_{1}(y)\right|(1+|y|)^{|\beta|}}{1-|y|} d y,
$$

where $\rho_{1}(y):=(n-1) \rho_{*}(|y|)+|y| \rho_{*}^{\prime}(|y|)$. 
By applying Lemma 2.2 to distance functions, the regularized distance for any closed set can be derived.

Definition 2.4 (Regularized distance) Let $S$ be a closed set in $\mathbb{R}^{n}$. For any given $\xi \in(0,1)$, there exists a function $\mathrm{RD}_{S, \xi} \in C^{\infty}\left(S^{c}\right)$ such that, for all $x \in S^{c}$,

$$
(1+\xi)^{-2} \operatorname{dist}(x, S) \leq \mathrm{RD}_{S, \xi}(x) \leq(1-\xi)^{-2} \operatorname{dist}(x, S)
$$

and

$$
\left|\frac{\partial^{\alpha}}{\partial x^{\alpha}} \mathrm{RD}_{S, \xi}(x)\right| \leq P_{\alpha}(\xi \operatorname{dist}(x, S))^{1-|\alpha|}, \quad \forall \alpha \in \mathbb{N}_{0}^{n} \text { with }|\alpha| \geq 1 .
$$

The function $\mathrm{RD}_{S, \xi}$ is called the regularized distance from $S$.

Next, we introduce two types of open sets.

Definition 2.5 (Special Lipschitz domain [12]) Let $\phi: \mathbb{R}^{n-1} \rightarrow \mathbb{R}(n=2,3, \ldots)$ be a function satisfying the Lipschitz condition, i.e., for some $M>0$,

$$
|\phi(x)-\phi(y)| \leq M|x-y|, \quad \forall x, y \in \mathbb{R}^{n-1} .
$$

Then, $\Omega$ is called a special Lipschitz domain if it is written as $\Omega:=\left\{\left(x^{\prime}, x_{n}\right) \in \mathbb{R}^{n}: x_{n}>\phi\left(x^{\prime}\right)\right\}$ with $x^{\prime}=\left(x_{1}, x_{2}, \ldots, x_{n-1}\right) \in \mathbb{R}^{n-1}$.

The positive number $M$ in Definition 2.5 is called the Lipschitz constant of $\Omega$. Generalizing the special Lipschitz domain, the domain with minimally smooth boundary is defined as follows.

Definition 2.6 (Domain with minimally smooth boundary [12]) An open set $\Omega \subset \mathbb{R}^{n}(n=$ $2,3, \ldots)$ is said to be a domain with minimally smooth boundary if there exist $\varepsilon>0, N \in \mathbb{N}$, $M>0$, and a sequence $\left\{U_{i}\right\}_{i \in \mathbb{N}}$ of open subsets of $\mathbb{R}^{n}$ such that

(1) for any $x \in \partial \Omega, B(x, \varepsilon) \subset U_{i}$ for some $i \in \mathbb{N}$;

(2) no point in $\mathbb{R}^{n}$ belongs to more than $N$ of $U_{i}$; and

(3) for any $i \in \mathbb{N}$, there exists a special Lipschitz domain $\Omega_{i}$, the Lipschitz bound of which is not more than $M$, such that $U_{i} \cap \Omega=U_{i} \cap \Omega_{i}$.

The positive number $M$ in Definition 2.6 is called the Lipschitz constant of $\Omega$, and $N$ in Definition 2.6 is called the overlap number of $\Omega$.

Remark 2.7 The domain with minimally smooth boundary defined in Definition 2.6 is referred to by various names. For example, it is called a domain satisfying the strong local Lipschitz condition in [18]. In addition, numerous researchers simply call it a Lipschitz domain, although several other definitions exist (see, e.g., Section 1.2.1 in [27]).

\section{Construction of extension operator}

Here, we describe Stein's construction method for extension operators [12]. Stein first constructed an extension operator on a special Lipschitz domain. He then expanded it to that on a domain with minimally smooth boundary. 


\subsection{Extension operator on special Lipschitz domain}

Let $\Omega^{\prime} \subset \mathbb{R}^{n}(n=2,3, \ldots)$ be a special Lipschitz domain, i.e., $\Omega^{\prime}$ is written in the form $\Omega^{\prime}:=\left\{\left(x^{\prime}, x_{n}\right) \in \mathbb{R}^{n}: x_{n}>\phi\left(x^{\prime}\right)\right\}, x^{\prime}=\left(x_{1}, x_{2}, \ldots, x_{n-1}\right) \in \mathbb{R}^{n-1}$, with a Lipschitz continuous function $\phi: \mathbb{R}^{n-1} \rightarrow \mathbb{R}$, the Lipschitz constant of which is $M_{\Omega^{\prime}}$. For given $\xi>0$, let $\mathrm{RD}_{\Omega^{\prime}, \xi}$ be the regularized distance with the bound $P_{\alpha}$ as in (9). Moreover, for given $\tau>0$, let us define $g_{\Omega^{\prime}, \tau, \xi}^{*}:=(1+\tau) C_{\Omega^{\prime}, \xi} \mathrm{RD}_{\Omega^{\prime}, \xi}$ with $C_{\Omega^{\prime}, \xi}:=(1+\xi)^{2} \sqrt{1+M_{\Omega^{\prime}}^{2}}$. Then, for any $k \in N_{0}$ and any $p \in[1, \infty)$, the operator $E_{\Omega^{\prime}, \tau, \xi}$, defined by

$$
\left(E_{\Omega^{\prime}, \tau, \xi} u\right)\left(x^{\prime}, x_{n}\right):= \begin{cases}u\left(x^{\prime}, x_{n}\right), & \forall\left(x^{\prime}, x_{n}\right) \in \overline{\Omega^{\prime}}, \\ \int_{1}^{\infty} u\left(x^{\prime}, x_{n}+t g_{\Omega^{\prime}, \tau, \xi}^{*}\left(x^{\prime}, x_{n}\right)\right) \psi(t) d t, & \forall\left(x^{\prime}, x_{n}\right) \in\left(\overline{\Omega^{\prime}}\right)^{c},\end{cases}
$$

becomes the extension operator from $W^{k, p}\left(\Omega^{\prime}\right)$ to $W^{k, p}\left(\mathbb{R}^{n}\right)$, where $\psi: \mathbb{R} \rightarrow \mathbb{R}$ is a function satisfying

$$
\int_{1}^{\infty} \psi(t) d t=1, \quad \int_{1}^{\infty} t^{m} \psi(t) d t=0, \quad \forall m \in \mathbb{N} .
$$

A concrete selection of the function $\psi$ can be found in (31). Note that since $(1+$ $\left.M_{\Omega^{\prime}}^{2}\right)^{-1 / 2} \operatorname{dist}\left(x,\left(\overline{\Omega^{\prime}}\right)^{c}\right) \geq \phi\left(x^{\prime}\right)-x_{n}$ for all $\left(x^{\prime}, x_{n}\right) \in\left(\overline{\Omega^{\prime}}\right)^{c}$, we have $g_{\Omega^{\prime}, \tau, \xi}^{*}\left(x^{\prime}, x_{n}\right) \geq(1+$ $\tau)\left(\phi\left(x^{\prime}\right)-x_{n}\right)$.

Remark 3.1 In the original construction method, Stein set $\tau$ and $\xi$ to concrete values [12]. However, since the selection of $\tau$ and $\xi$ affects the accuracy of estimation of the embedding constants, we do not fix them above. Their selection will be discussed later.

\subsection{Extension operator on domain with minimally smooth boundary}

Let $\Omega$ be a domain with minimally smooth boundary. Let $\left\{U_{i}\right\}_{i \in \mathbb{N}}$ be the sequence as in Definition 2.6. Let $\varepsilon$ be a positive number such that $U_{i}^{\frac{3}{4} \varepsilon}$ are not empty for all $i \in \mathbb{N}$, and if $\operatorname{dist}(x, \partial \Omega) \leq \varepsilon / 2$, then $x \in U_{i}^{\frac{1}{2} \varepsilon}$ for some $i \in \mathbb{N}$. Let $\rho$ be any given mollifier defined as in Definition 2.1, and put $\rho_{\varepsilon}(x):=\varepsilon^{-n} \rho\left(x \varepsilon^{-1}\right)$. Let $\chi_{i}$ be the characteristic function of $U_{i}^{\frac{3}{4} \varepsilon}$, and put $\lambda_{i}^{\varepsilon}(x):=\left(\chi_{i} * \rho_{\frac{1}{4} \varepsilon}\right)(x)$. Put

$$
\begin{aligned}
& U_{0}=\left\{x \in \mathbb{R}^{n}: \operatorname{dist}(x, \Omega)<\frac{1}{4} \varepsilon\right\}, \\
& U_{+}=\left\{x \in \mathbb{R}^{n}: \operatorname{dist}(x, \partial \Omega)<\frac{3}{4} \varepsilon\right\},
\end{aligned}
$$

and

$$
U_{-}=\left\{x \in \Omega: \operatorname{dist}(x, \partial \Omega)>\frac{1}{4} \varepsilon\right\} .
$$

Let $\chi_{0}, \chi_{+}$, and $\chi_{-}$be the corresponding characteristic functions of $U_{0}, U_{+}$, and $U_{-}$, respectively. Let $\lambda_{0}^{\varepsilon}:=\chi_{0} * \rho_{\frac{1}{4} \varepsilon}, \lambda_{+}^{\varepsilon}:=\chi_{+} * \rho_{\frac{1}{4} \varepsilon}$, and $\lambda_{-}^{\varepsilon}:=\chi_{-} * \rho_{\frac{1}{4} \varepsilon}$. Put

$$
\Lambda_{+}^{\varepsilon}:=\lambda_{0}^{\varepsilon} \frac{\lambda_{+}^{\varepsilon}}{\lambda_{+}^{\varepsilon}+\lambda_{-}^{\varepsilon}} \quad \text { and } \quad \Lambda_{-}^{\varepsilon}:=\lambda_{0}^{\varepsilon} \frac{\lambda_{-}^{\varepsilon}}{\lambda_{+}^{\varepsilon}+\lambda_{-}^{\varepsilon}} \text {. }
$$


To each $U_{i}$, there corresponds a special Lipschitz domain $\Omega_{i}$ as in Definition 2.6. Let $E_{\Omega_{i}, \tau, \xi}^{i}$ be the extension operator for each $\Omega_{i}$ constructed by (10). For any $k \in N_{0}$ and any $p \in$ $[1, \infty)$, the following operator $E_{\Omega, \tau, \xi, \varepsilon}$ becomes the extension operator from $W^{k, p}(\Omega)$ to $W^{k, p}\left(\mathbb{R}^{n}\right)$ :

$$
\left(E_{\Omega, \tau, \xi, \varepsilon} u\right)(x):=\Lambda_{+}^{\varepsilon}(x)\left(\frac{\sum_{i=1}^{\infty} \lambda_{i}^{\varepsilon}(x) E_{\Omega_{i} \tau, \xi}^{i}\left(\lambda_{i}^{\varepsilon} u\right)(x)}{\sum_{i=1}^{\infty} \lambda_{i}^{\varepsilon}(x)^{2}}\right)+\Lambda_{-}^{\varepsilon}(x) u(x)
$$

for all $x \in \mathbb{R}^{n}$.

Here, one can observe that

- supp $\lambda_{i}^{\varepsilon} \subset U_{i}$, and $\lambda_{i}^{\varepsilon}(x)=1$ if $x \in U_{i}^{\frac{1}{2} \varepsilon}$;

- if $x \in \operatorname{supp} \Lambda_{+}^{\varepsilon}$, then $\sum_{i \in \mathbb{N}} \lambda_{i}^{\varepsilon}(x) \geq 1$;

- the bounds of the derivatives of $\lambda_{i}^{\varepsilon}$ are independent of $i$ and depend only on the $L^{1}$ norm of the corresponding derivatives of $\rho_{\frac{1}{4}} \varepsilon$;

- $\lambda_{0}^{\varepsilon}(x)=1$ if $x \in \bar{\Omega}$

- $\lambda_{+}^{\varepsilon}(x)=1$ if $\operatorname{dist}(x, \partial \Omega) \leq \varepsilon / 2$;

- $\lambda_{-}^{\varepsilon}(x)=1$ if $x \in \Omega$ and $\operatorname{dist}(x, \partial \Omega) \geq \varepsilon / 2$;

- the supports of $\lambda_{0}^{\varepsilon}, \lambda_{+}^{\varepsilon}$, and $\lambda_{-}^{\varepsilon}$ are contained in the $\varepsilon / 2$-neighborhood of $\Omega$, in the $\varepsilon$-neighborhood of $\partial \Omega$, and in $\Omega$, respectively;

- the functions $\lambda_{0}^{\varepsilon}, \lambda_{+}^{\varepsilon}$, and $\lambda_{-}^{\varepsilon}$ are bounded in $\mathbb{R}^{n}$, and all their partial derivatives are also bounded;

- all the derivatives of $\Lambda_{+}^{\varepsilon}$ and $\Lambda_{-}^{\varepsilon}$ are bounded on $\mathbb{R}^{n}$;

- $\Lambda_{+}^{\varepsilon}+\Lambda_{-}^{\varepsilon}$ is 1 on $\bar{\Omega}$ and is 0 outside the $\varepsilon / 2$-neighborhood of $\Omega$.

Remark 3.2 In Stein's original construction method, $\varepsilon>0$ is assumed to be sufficiently small [12]. However, since the bounds for the derivatives of $\lambda_{i}^{\varepsilon}$ increase as $\varepsilon$ decreases, a small $\varepsilon$ makes the norm of the corresponding extension operator large. Therefore, we should select the value of $\varepsilon$ by taking this property into consideration. The selection of $\varepsilon$ for concrete domains $\Omega$ will be discussed in Section 5.2.

\section{Formula for estimating operator norm}

Let us first present the following lemma, which gives bounds for the operator norm of the extension operator on special Lipschitz domains constructed by the method in Section 3.1.

Lemma 4.1 For a special Lipschitz domain $\Omega^{\prime} \subset \mathbb{R}^{n}(n=2,3, \ldots)$, let $E\left(=E_{\Omega^{\prime}, \tau, \xi}\right)$ be the extension operator constructed by (10). Then,

$$
\|E u\|_{L^{p}\left(\mathbb{R}^{n}\right)} \leq A_{p, \tau, \xi}\left(\Omega^{\prime}\right)\|u\|_{L^{p}\left(\Omega^{\prime}\right)}, \quad \forall u \in L^{p}\left(\Omega^{\prime}\right)
$$

and

$$
\|\nabla(E u)\|_{L^{p}\left(\mathbb{R}^{n)}\right.} \leq A_{p, \tau, \xi}^{\prime}\left(\Omega^{\prime}\right)\|\nabla u\|_{L^{p}\left(\Omega^{\prime}\right)}, \quad \forall u \in W^{1, p}\left(\Omega^{\prime}\right),
$$

for

$$
A_{p, \tau, \xi}\left(\Omega^{\prime}\right)=\left\{\left(A_{0} Q\right)^{p}+1\right\}^{1 / p}
$$


and

$$
A_{p, \tau, \xi}^{\prime}\left(\Omega^{\prime}\right)=\max \left\{\left[2^{p-1}\left(A_{0} Q\right)^{p}+1\right]^{1 / p},\left[(n-1) 2^{p-1}(B Q)^{p}+\left\{\left(A_{0}+B\right) Q\right\}^{p}+1\right]^{1 / p}\right\},
$$

respectively. Here,

- $A_{0}$ and $A_{1}$ are constants satisfying $|\psi(t)| \leq A_{0} / t^{2}(t \geq 1)$ and $|\psi(t)| \leq A_{1} / t^{3}(t \geq 1)$, respectively;

- P corresponds to $P_{\alpha}$ with $|\alpha|=1$;

- Q and B are defined as

$$
Q\left(=Q_{\Omega^{\prime}, \tau, \xi, p}\right):=\frac{p(1+\tau)(1+\xi)^{2}}{(p+1) \tau^{1+1 / p}(1-\xi)^{2}} \sqrt{1+M_{\Omega^{\prime}}^{2}}
$$

and

$$
B\left(=B_{\Omega^{\prime}, \xi, \tau}\right):=A_{1} P(1+\xi)^{2}(1+\tau) \sqrt{1+M_{\Omega^{\prime}}^{2}}
$$

Proof Since $C^{\infty}\left(\Omega^{\prime}\right)$ is dense in $W^{1, p}\left(\Omega^{\prime}\right)$, it suffices to consider $u \in C^{\infty}\left(\Omega^{\prime}\right)$. Moreover, $\Omega^{\prime}$ is written in the form $\Omega^{\prime}:=\left\{\left(x^{\prime}, x_{n}\right) \in \mathbb{R}^{n}: x_{n}>\phi\left(x^{\prime}\right)\right\}, x^{\prime}=\left(x_{1}, x_{2}, \ldots, x_{n-1}\right) \in \mathbb{R}^{n-1}$, with a Lipschitz continuous function $\phi: \mathbb{R}^{n-1} \rightarrow \mathbb{R}$, the Lipschitz constant of which is $M_{\Omega^{\prime}}$. Hereafter, for simplicity, we write $u_{y}=\partial_{y} u, g^{*}=g_{\Omega^{\prime}, \tau, \xi}^{*}, g_{y}^{*}=\partial_{y} g^{*}$.

First step: estimating $A_{p, \tau, \xi}\left(\Omega^{\prime}\right)$

If $y<\phi(x)$ with $y \in \mathbb{R}$ and $x=\left(x_{1}, x_{2}, \ldots, x_{n-1}\right) \in \mathbb{R}^{n-1}$, then

$$
\begin{aligned}
|(E u)(x, y)| & =\left|\int_{1}^{\infty} u\left(x, y+\operatorname{tg}^{*}(x, y)\right) \psi(t) d t\right| \\
& \leq A_{0} \int_{1}^{\infty}\left|u\left(x, y+\operatorname{tg}^{*}(x, y)\right)\right| \frac{d t}{t^{2}} .
\end{aligned}
$$

Moreover, we have $g^{*}(x, y) \geq(1+\tau)(\phi(x)-y)=(1+\tau)|y-\phi(x)|$. In addition, it follows that $\phi(x)-y \geq \operatorname{dist}\left((x, y), \overline{\Omega^{\prime}}\right)$ for all $x \in \mathbb{R}^{n-1}$ and $y \in \mathbb{R}$. Since $\operatorname{dist}\left((x, y), \overline{\Omega^{\prime}}\right) \geq(1-$ $\xi)^{2} \mathrm{RD}_{\Omega^{\prime}, \xi}(x, y)$, it follows that

$$
\begin{aligned}
|y-\phi(x)|= & \geq \operatorname{dist}\left((x, y), \overline{\Omega^{\prime}}\right) \\
& \geq(1-\xi)^{2} \operatorname{RD}_{\Omega^{\prime}, \xi}(x, y) \\
& =(1-\xi)^{2}(1+\tau)^{-1} C_{\Omega^{\prime}, \xi}^{-1} g^{*}(x, y) .
\end{aligned}
$$

Now, recall that $g^{*}=(1+\tau) C_{\Omega^{\prime}, \xi} \mathrm{RD}_{\Omega^{\prime}, \xi}$. From (16) we obtain $g^{*}(x, y) \leq a|y-\phi(x)|$, where $a\left(=a_{\Omega^{\prime}, \tau, \xi}\right):=(1+\tau)(1+\xi)^{2}(1-\xi)^{-2} \sqrt{1+M_{\Omega^{\prime}}^{2}}$. Putting $s=y-\phi(x)+\operatorname{tg}^{*}(x, y)$, it follows from (15) that

$$
\begin{aligned}
|(E u)(x, y)| & \leq A_{0} \int_{1}^{\infty}\left|u\left(x, y+\operatorname{tg}^{*}(x, y)\right)\right| \frac{d t}{t^{2}} \\
& =A_{0} g^{*}(x, y) \int_{y-\phi(x)+g^{*}(x, y)}^{\infty}|u(x, s+\phi(x))|(s-(y-\phi(x)))^{-2} d s \\
& \leq A_{0} a|y-\phi(x)| \int_{\tau|y-\phi(x)|}^{\infty}|u(x, s+\phi(x))| s^{-2} d s .
\end{aligned}
$$


Kanaka et al. Journal of Inequalities and Applications <wide>(2015<wide>) 2015:389

Page 8 of 23

By changing the integration variable as $\tau(y-\phi(x))=w$, we have

$$
\begin{aligned}
& \int_{-\infty}^{\phi(x)}|(E u)(x, y)|^{p} d y \\
& \quad \leq\left(\frac{a A_{0}}{\tau}\right)^{p} \int_{-\infty}^{\phi(x)}\left(\tau|y-\phi(x)| \int_{\tau|y-\phi(x)|}^{\infty}|u(x, s+\phi(x))| s^{-2} d s\right)^{p} d y, \\
& \quad=\left(\frac{a A_{0}}{\tau^{1+1 / p}}\right)^{p} \int_{-\infty}^{0}\left(|w| \int_{|w|}^{\infty}|u(x, s+\phi(x))| s^{-2} d s\right)^{p} d w \\
& \quad=\left(\frac{a A_{0}}{\tau^{1+1 / p}}\right)^{p} \int_{0}^{\infty}\left(\int_{|w|}^{\infty}|u(x, s+\phi(x))| s^{-2} d s\right)^{p}|w|^{(p+1)-1} d w .
\end{aligned}
$$

Hardy's inequality, which can be found in Lemma C.1, gives

$$
\begin{aligned}
\int_{-\infty}^{\phi(x)}|(E u)(x, y)|^{p} d y & \leq\left(\frac{p A_{0} a}{(p+1) \tau^{1+1 / p}}\right)^{p} \int_{0}^{\infty}\left(|u(x, s+\phi(x))| s^{-1}\right)^{p} s^{p} d s \\
& =\left(\frac{p A_{0} a}{(p+1) \tau^{1+1 / p}}\right)^{p} \int_{0}^{\infty}|u(x, s+\phi(x))|^{p} d s \\
& =\left(\frac{p A_{0} a}{(p+1) \tau^{1+1 / p}}\right)^{p} \int_{\phi(x)}^{\infty}|u(x, y)|^{p} d y .
\end{aligned}
$$

Moreover, from the definition (10) of the extension operator we have

$$
\int_{\phi(x)}^{\infty}|(E u)(x, y)|^{p} d y=\int_{\phi(x)}^{\infty}|u(x, y)|^{p} d y .
$$

From (17) and (18) it follows that

$$
\left(\int_{-\infty}^{\infty}|(E u)(x, y)|^{p} d y\right)^{1 / p} \leq\left\{\left(A_{0} Q\right)^{p}+1\right\}^{1 / p}\left(\int_{\phi(x)}^{\infty}|u(x, y)|^{p} d y\right)^{1 / p},
$$

where $Q\left(=Q_{\Omega^{\prime}, \tau, \xi, p}\right):=p a_{\Omega^{\prime}, \tau, \xi} /\left\{(p+1) \tau^{1+1 / p}\right\}$. Integrating both sides of (19) by $x$, we find that (13) holds for

$$
A_{p, \tau, \xi}\left(\Omega^{\prime}\right)=\left\{\left(A_{0} Q\right)^{p}+1\right\}^{1 / p}
$$

Second step: estimating $A_{p, \tau, \xi}^{\prime}\left(\Omega^{\prime}\right)$

Inequality (9) ensures that $\left|g_{x_{j}}^{*}(x, y)\right| \leq B / A_{1}$ for $j \in\{1,2, \ldots, n\}$. If $y<\phi(x)$ with $y \in \mathbb{R}$ and $x=\left(x_{1}, x_{2}, \ldots, x_{n-1}\right) \in \mathbb{R}^{n-1}$, then

$$
\begin{aligned}
\partial_{y}(E u)(x, y)= & \partial_{y} \int_{1}^{\infty} u\left(x, y+\operatorname{tg}^{*}(x, y)\right) \psi(t) d t \\
= & \int_{1}^{\infty} u_{y}\left(x, y+\operatorname{tg}^{*}(x, y)\right)\left(1+\operatorname{tg}_{y}^{*}(x, y)\right) \psi(t) d t \\
= & \int_{1}^{\infty} u_{y}\left(x, y+\operatorname{tg}^{*}(x, y)\right) \psi(t) d t \\
& +g_{y}^{*}(x, y) \int_{1}^{\infty} u_{y}\left(x, y+\operatorname{tg}^{*}(x, y)\right) t \psi(t) d t .
\end{aligned}
$$


Therefore, we have

$$
\begin{aligned}
& \left|\partial_{y}(E u)(x, y)\right| \\
& \leq\left|\int_{1}^{\infty} u_{y}\left(x, y+\operatorname{tg}^{*}(x, y)\right) \psi(t) d t\right| \\
& \quad+\left|g_{y}^{*}(x, y)\right|\left|\int_{1}^{\infty} u_{y}\left(x, y+\operatorname{tg}^{*}(x, y)\right) t^{3} \psi(t) \frac{d t}{t^{2}}\right| \\
& \leq\left(A_{0}+B\right) \int_{1}^{\infty}\left|u_{y}\left(x, y+\operatorname{tg}^{*}(x, y)\right)\right| \frac{d t}{t^{2}}, \quad y<\phi(x) .
\end{aligned}
$$

From the similar discussion in the first step we have

$$
\int_{-\infty}^{\infty}\left|\partial_{y}(E u)(x, y)\right|^{p} d y \leq\left[\left\{\left(A_{0}+B\right) Q\right\}^{p}+1\right] \int_{\phi(x)}^{\infty}\left|u_{y}(x, y)\right|^{p} d y .
$$

On the other hand, for $j \in\{1,2, \ldots, n-1\}$ and $y<\phi(x)$,

$$
\begin{aligned}
\partial_{x_{j}}(E u)(x, y) \\
=\partial_{x_{j}} \int_{1}^{\infty} u\left(x, y+\operatorname{tg}^{*}(x, y)\right) \psi(t) d t \\
=\int_{1}^{\infty}\left\{u_{x_{j}}\left(x, y+\operatorname{tg}^{*}(x, y)\right)+u_{y}\left(x, y+\operatorname{tg}^{*}(x, y)\right) \operatorname{tg}_{x_{j}}^{*}(x, y)\right\} \psi(t) d t \\
=\int_{1}^{\infty} u_{x_{j}}\left(x, y+\operatorname{tg}^{*}(x, y)\right) \psi(t) d t \\
\quad+g_{x_{j}}^{*}(x, y) \int_{1}^{\infty} u_{y}\left(x, y+\operatorname{tg}^{*}(x, y)\right) t \psi(t) d t .
\end{aligned}
$$

Therefore, we have

$$
\begin{aligned}
\left|\partial_{x_{j}}(E u)(x, y)\right| & \\
\leq & \left|\int_{1}^{\infty} u_{x_{j}}\left(x, y+\operatorname{tg}^{*}(x, y)\right) \psi(t) d t\right| \\
& +\left|g_{x_{j}}^{*}(x, y)\right|\left|\int_{1}^{\infty} u_{y}\left(x, y+\operatorname{tg}^{*}(x, y)\right) t \psi(t) d t\right| \\
\leq & A_{0} \int_{1}^{\infty}\left|u_{x_{j}}\left(x, y+\operatorname{tg}^{*}(x, y)\right)\right| \frac{d t}{t^{2}} \\
& +B \int_{1}^{\infty}\left|u_{y}\left(x, y+\operatorname{tg}^{*}(x, y)\right)\right| \frac{d t}{t^{2}}, \quad y<\phi(x) .
\end{aligned}
$$

Since $(s+t)^{p} \leq 2^{p-1}\left(s^{p}+t^{p}\right)$ for $s, t>0$ and $p>1$, it follows from the similar discussion in (17) that

$$
\begin{aligned}
& \int_{-\infty}^{\phi(x)}\left|\partial_{x_{j}}(E u)(x, y)\right|^{p} d y \\
& \quad \leq 2^{p-1}\left(A_{0} Q\right)^{p} \int_{\phi(x)}^{\infty}\left|u_{x_{j}}(x, y)\right|^{p} d y+2^{p-1}(B Q)^{p} \int_{\phi(x)}^{\infty}\left|u_{y}(x, y)\right|^{p} d y .
\end{aligned}
$$


Therefore,

$$
\begin{aligned}
\int_{-\infty}^{\infty}\left|\partial_{x_{j}}(E u)(x, y)\right|^{p} d y \leq & \left\{2^{p-1}\left(A_{0} Q\right)^{p}+1\right\} \int_{\phi(x)}^{\infty}\left|u_{x_{j}}(x, y)\right|^{p} d y \\
& +2^{p-1}(B Q)^{p} \int_{\phi(x)}^{\infty}\left|u_{y}(x, y)\right|^{p} d y
\end{aligned}
$$

for $j \in\{1,2, \ldots, n-1\}$. From (20) and (21) we have

$$
\begin{aligned}
\sum_{j=1}^{n} & \int_{-\infty}^{\infty}\left|\partial_{x_{j}}(E u)(x, y)\right|^{p} d y \\
= & \sum_{j=1}^{n-1} \int_{-\infty}^{\infty}\left|\partial_{x_{j}}(E u)(x, y)\right|^{p} d y+\int_{-\infty}^{\infty}\left|\partial_{y}(E u)(x, y)\right|^{p} d y \\
\leq & \left\{2^{p-1}\left(A_{0} Q\right)^{p}+1\right\} \sum_{j=1}^{n-1} \int_{\phi(x)}^{\infty}\left|u_{x_{j}}(x, y)\right|^{p} d y \\
& +(n-1) 2^{p-1}(B Q)^{p} \int_{\phi(x)}^{\infty}\left|u_{y}(x, y)\right|^{p} d y \\
& +\left[\left\{\left(A_{0}+B\right) Q\right\}^{p}+1\right] \int_{\phi(x)}^{\infty}\left|u_{y}(x, y)\right|^{p} d y \\
= & \left\{2^{p-1}\left(A_{0} Q\right)^{p}+1\right\} \sum_{j=1}^{n-1} \int_{\phi(x)}^{\infty}\left|u_{x_{j}}(x, y)\right|^{p} d y \\
& +\left[(n-1) 2^{p-1}(B Q)^{p}+\left\{\left(A_{0}+B\right) Q\right\}^{p}+1\right] \int_{\phi(x)}^{\infty}\left|u_{y}(x, y)\right|^{p} d y .
\end{aligned}
$$

This ensures that inequality (14) holds for

$$
\begin{aligned}
& A_{p, \tau, \xi}^{\prime}\left(\Omega^{\prime}\right) \\
& \quad=\max \left\{\left[2^{p-1}\left(A_{0} Q\right)^{p}+1\right]^{1 / p},\left[(n-1) 2^{p-1}(B Q)^{p}+\left\{\left(A_{0}+B\right) Q\right\}^{p}+1\right]^{1 / p}\right\} .
\end{aligned}
$$

The following formula enables us to estimate the operator norm $A_{q}(\Omega)$ for the extension operator on domains with minimally smooth boundary constructed by the method described in Section 3.

Theorem 4.2 For a domain $\Omega \subset \mathbb{R}^{n}(n=2,3, \ldots)$ with minimally smooth boundary, let $E$ $\left(=E_{\Omega, \tau, \xi, \varepsilon}\right)$ be the extension operator constructed by (12). Then, letting $\gamma$ be a given positive number, we have

$$
\|\nabla(E u)\|_{L^{p}\left(\mathbb{R}^{n)}\right.} \leq A_{p}(\Omega)\left(\|\nabla u\|_{L^{p}(\Omega)}+\gamma\|u\|_{L^{p}(\Omega)}\right), \quad \forall u \in W^{1, p}(\Omega),
$$

with

$$
A_{p}(\Omega)= \begin{cases}N A^{\prime}+1, & R \leq \gamma \\ b_{\varepsilon}\left(4 N A+2 N^{3 / 2} A+N A^{\prime}+3\right) n^{1 / p} / \gamma, & R>\gamma\end{cases}
$$


where $N$ is the overlap number of $\Omega, b_{\varepsilon}$ is a positive number satisfying $b_{\varepsilon} \geq \int_{\mathbb{R}^{n}}\left|\partial_{x_{j}} \rho_{\frac{1}{4} \varepsilon}(x)\right| d x$ for all $j \in\{1,2, \ldots, n\}$, and $R:=b_{\varepsilon}\left(4 N A+2 N^{3 / 2} A+N A^{\prime}+3\right) n^{1 / p} /\left(N A^{\prime}+1\right)$. The constants $A$ and $A^{\prime}$ are determined by $A=\sup \left\{A_{p, \tau, \xi}\left(\Omega_{i}\right): i \in \mathbb{N}\right\}$ and $A^{\prime}=\sup \left\{A_{p, \tau, \xi}^{\prime}\left(\Omega_{i}\right): i \in \mathbb{N}\right\}$ for the operator norms $A_{p, \tau, \xi}\left(\Omega_{i}\right)$ and $A_{p, \tau, \xi}^{\prime}\left(\Omega_{i}\right)$ of $E^{i}\left(=E_{\Omega_{i, \tau}, \xi}^{i}\right)$ satisfying (13) and (14) with the notational replacement $\Omega^{\prime}=\Omega_{i}$, respectively.

Proof For any $j \in\{1,2, \ldots, n\}$, we have

$$
\left|\partial_{x_{j}} \lambda_{i}^{\varepsilon}\right| \leq \int_{\mathbb{R}^{n}}\left|\partial_{x_{j}} \rho_{\frac{1}{4} \varepsilon}(x)\right| d x \leq b_{\varepsilon} ;
$$

this bound does not depend on the index $i$. Likewise, $\left|\partial_{x_{j}} \lambda_{0}^{\varepsilon}\right|,\left|\partial_{x_{j}} \lambda_{+}^{\varepsilon}\right|$, and $\left|\partial_{x_{j}} \lambda_{-}^{\varepsilon}\right|$ are bounded by $b_{\varepsilon}$. Moreover,

$$
\begin{aligned}
\left|\partial_{x_{j}} \Lambda_{+}^{\varepsilon}\right| & =\left|\left(\partial_{x_{j}} \lambda_{0}^{\varepsilon}\right) \frac{\lambda_{+}^{\varepsilon}}{\lambda_{+}^{\varepsilon}+\lambda_{-}^{\varepsilon}}+\lambda_{0}^{\varepsilon} \frac{\left(\partial_{x_{j}} \lambda_{+}^{\varepsilon}\right)\left(\lambda_{+}^{\varepsilon}+\lambda_{-}^{\varepsilon}\right)-\lambda_{+}^{\varepsilon}\left(\partial_{x_{j}} \lambda_{+}^{\varepsilon}+\partial_{x_{j}} \lambda_{-}^{\varepsilon}\right)}{\left(\lambda_{+}^{\varepsilon}+\lambda_{-}^{\varepsilon}\right)^{2}}\right| \\
& \leq 3 b_{\varepsilon}=: b_{+} .
\end{aligned}
$$

It is easily confirmed that $\left|\partial_{x_{j}} \Lambda_{-}^{\varepsilon}\right|$ is also bounded by $3 b_{\varepsilon}=: b_{-}$(we distinguish $b_{+}$and $b_{-}$to avoid confusion in the following proof). Hereafter, we simply denote $\bigcup_{i} U_{i}^{\varepsilon / 2}$ by $U^{*}, \sum_{i \in \mathbb{N}}$ by $\sum, \lambda_{i}^{\varepsilon}$ by $\lambda_{i}, \Lambda_{+}^{\varepsilon}$ by $\Lambda_{+}$, and $\Lambda_{-}^{\varepsilon}$ by $\Lambda_{-}$. For $u \in W^{1, p}(\Omega)$,

$$
\begin{aligned}
\|\nabla(E u)\|_{L^{p}\left(\mathbb{R}^{n}\right)} & \left(\sum_{j} \int_{\mathbb{R}^{n}}\left|\partial_{x_{j}}(E u)\right|^{p} d x\right)^{1 / p} \\
\leq & \left(\sum_{j} \int_{\mathbb{R}^{n}}\left|\left(\partial_{x_{j}} \Lambda_{+}\right)\left(\frac{\sum \lambda_{i} E^{i}\left(\lambda_{i} u\right)}{\sum \lambda_{i}^{2}}\right)\right|^{p} d x\right)^{1 / p}+\left(\sum_{j} \int_{\mathbb{R}^{n}}\left|\Lambda_{+}(o)\right|^{p} d x\right)^{1 / p} \\
& +\left(\sum_{j} \int_{\mathbb{R}^{n}}\left|\left(\partial_{x_{j}} \Lambda_{-}\right) u\right|^{p} d x\right)^{1 / p}+\left(\sum_{j} \int_{\mathbb{R}^{n}}\left|\Lambda_{-}\left(\partial_{x_{j}} u\right)\right|^{p} d x\right)^{1 / p},
\end{aligned}
$$

where

$$
\circ:=\frac{\left(\partial_{x_{j}} \sum \lambda_{i} E^{i}\left(\lambda_{i} u\right)\right)\left(\sum \lambda_{i}^{2}\right)-\left(\sum \lambda_{i} E^{i}\left(\lambda_{i} u\right)\right)\left(\partial_{x_{j}} \sum \lambda_{i}^{2}\right)}{\left(\sum \lambda_{i}^{2}\right)^{2}} .
$$

From Lemma C.2, the first term of (25) is evaluated as

$$
\begin{aligned}
& \left(\sum_{j} \int_{\mathbb{R}^{n}}\left|\left(\partial_{x_{j}} \Lambda_{+}\right)\left(\frac{\sum \lambda_{i} E^{i}\left(\lambda_{i} u\right)}{\sum \lambda_{i}^{2}}\right)\right|^{p} d x\right)^{1 / p} \\
& \leq b_{+}\left(\sum_{j} \int_{U^{*}}\left|\frac{\sum \lambda_{i} E^{i}\left(\lambda_{i} u\right)}{\sum \lambda_{i}^{2}}\right|^{p} d x\right)^{1 / p} \\
& \leq b_{+} n^{1 / p}\left(\int_{U^{*}}\left|\sum \lambda_{i} E^{i}\left(\lambda_{i} u\right)\right|^{p} d x\right)^{1 / p} \\
& \leq b_{+} N^{1-1 / p} n^{1 / p}\left(\sum \int_{U_{i}}\left|E^{i}\left(\lambda_{i} u\right)\right|^{p} d x\right)^{1 / p}
\end{aligned}
$$




$$
\begin{aligned}
& \leq b_{+} N^{1-1 / p} A n^{1 / p}\left(\sum \int_{\Omega}\left|\lambda_{i} u\right|^{p} d x\right)^{1 / p} \\
& \leq b_{+} N A n^{1 / p}\left(\int_{\Omega}|u|^{p} d x\right)^{1 / p} .
\end{aligned}
$$

The second term of (25) is evaluated as

$$
\begin{aligned}
& \left(\sum_{j} \int_{\mathbb{R}^{n}}\left|\Lambda_{+}(\circ)\right|^{p} d x\right)^{1 / p} \\
& \leq\left(\sum_{j} \int_{U^{*}}\left|\frac{\partial_{x_{j}} \sum \lambda_{i} E^{i}\left(\lambda_{i} u\right)}{\sum \lambda_{i}^{2}}\right|^{p} d x\right)^{1 / p} \\
& \quad+\left(\sum_{j} \int_{U^{*}}\left|\frac{\left(\sum \lambda_{i} E^{i}\left(\lambda_{i} u\right)\right)\left(\partial_{x_{j}} \sum \lambda_{i}^{2}\right)}{\left(\sum \lambda_{i}^{2}\right)^{2}}\right|^{p} d x\right)^{1 / p} .
\end{aligned}
$$

The first term of (26) is evaluated as

$$
\begin{aligned}
& \left(\sum_{j} \int_{U^{*}}\left|\frac{\partial_{x_{j}} \sum \lambda_{i} E^{i}\left(\lambda_{i} u\right)}{\sum \lambda_{i}^{2}}\right|^{p} d x\right)^{1 / p} \\
& =\left(\sum_{j} \int_{U^{*}}\left|\frac{\sum\left(\partial_{x_{j}} \lambda_{i}\right) E^{i}\left(\lambda_{i} u\right)+\sum \lambda_{i}\left(\partial_{x_{j}} E^{i}\left(\lambda_{i} u\right)\right)}{\sum \lambda_{i}^{2}}\right|^{p} d x\right)^{1 / p} \\
& \leq\left(\sum_{j} \int_{U^{*}}\left|\sum\left(\partial_{x_{j}} \lambda_{i}\right) E^{i}\left(\lambda_{i} u\right)\right|^{p} d x\right)^{1 / p} \\
& \quad+\left(\sum_{j} \int_{U^{*}}\left|\sum \lambda_{i}\left(\partial_{x_{j}} E^{i}\left(\lambda_{i} u\right)\right)\right|^{p} d x\right)^{1 / p} .
\end{aligned}
$$

The first term of (27) is evaluated as

$$
\begin{aligned}
& \left(\sum_{j} \int_{U^{*}}\left|\sum_{i}\left(\partial_{x_{j}} \lambda_{i}\right) E^{i}\left(\lambda_{i} u\right)\right|^{p} d x\right)^{1 / p} \\
& \leq\left(\sum_{j} N^{p-1} \sum_{i} \int_{U^{*}}\left|\left(\partial_{x_{j}} \lambda_{i}\right) E^{i}\left(\lambda_{i} u\right)\right|^{p} d x\right)^{1 / p} \\
& \leq N^{1-1 / p}\left(\sum_{j} \sum_{i} \int_{U_{i}} b_{\varepsilon}^{p}\left|E^{i}\left(\lambda_{i} u\right)\right|^{p} d x\right)^{1 / p} \\
& \leq b_{\varepsilon} N^{1-1 / p}\left(\sum_{j} \sum_{i} \int_{U_{i}}\left|E^{i}\left(\lambda_{i} u\right)\right|^{p} d x\right)^{1 / p} \\
& \leq b_{\varepsilon} N^{1-1 / p} n^{1 / p}\left(\sum_{i} \int_{U_{i}}\left|E^{i}\left(\lambda_{i} u\right)\right|^{p} d x\right)^{1 / p} \\
& \leq b_{\varepsilon} N^{1-1 / p} A n^{1 / p}\left(\sum_{i} \int_{\Omega}\left|\lambda_{i} u\right|^{p} d x\right)^{1 / p} \\
& \leq b_{\varepsilon} N A n^{1 / p}\left(\int_{\Omega}|u|^{p} d x\right)^{1 / p} \cdot
\end{aligned}
$$


The second term of (27) is evaluated as

$$
\begin{aligned}
\left(\sum_{j} \int_{U^{*}}\left|\sum_{i} \lambda_{i}\left(\partial_{x_{j}} E^{i}\left(\lambda_{i} u\right)\right)\right|^{p} d x\right)^{1 / p} \\
\leq\left(\sum_{j} N^{p-1} \sum_{i} \int_{U^{*}}\left|\lambda_{i}\left(\partial_{x_{j}} E^{i}\left(\lambda_{i} u\right)\right)\right|^{p} d x\right)^{1 / p} \\
\leq N^{1-1 / p}\left(\sum_{i} \sum_{j} \int_{U_{i}}\left|\partial_{x_{j}} E^{i}\left(\lambda_{i} u\right)\right|^{p} d x\right)^{1 / p} \\
\leq N^{1-1 / p} A^{\prime}\left(\sum_{i} \sum_{j} \int_{\Omega}\left|\partial_{x_{j}}\left(\lambda_{i} u\right)\right|^{p} d x\right)^{1 / p} \\
=N^{1-1 / p} A^{\prime}\left(\sum_{i} \sum_{j} \int_{\Omega}\left|\left(\partial_{x_{j}} \lambda_{i}\right) u+\lambda_{i}\left(\partial_{x_{j}} u\right)\right|^{p} d x\right)^{1 / p} \\
\leq N^{1-1 / p} A^{\prime}\left(\sum_{i} \int_{\Omega} \sum_{j}\left|\left(\partial_{x_{j}} \lambda_{i}\right) u\right|^{p} d x\right)^{1 / p} \\
\leq N A^{\prime}\left\{b_{\varepsilon} n^{1 / p}\left(\int_{\Omega}|u|^{p} d x\right)^{1 / p}+\left(\int_{\Omega} \sum_{j}\left|\partial_{x_{j}} u\right|^{p} d x\right)^{1 / p}\right\} \\
\leq N A^{\prime}\left\{\left(\sum_{j} b_{\varepsilon}^{p} \int_{\Omega}|u|^{p} d x\right)^{1 / p}+\left(\int_{\Omega} \sum_{j}\left|\partial_{x_{j}} u\right|^{p} d x\right)^{1 / p}\right\}
\end{aligned}
$$

Since Hölder's inequality ensures that $\left|\sum\left(\partial_{x_{j}} \lambda_{i}\right) \lambda_{i}\right| \leq b_{\varepsilon} N^{1 / 2}\left(\sum \lambda_{i}^{2}\right)^{1 / 2}$, the second term of (26) is evaluated as

$$
\begin{aligned}
& \left(\sum_{j} \int_{U^{*}}\left|\frac{\left(\sum \lambda_{i} E^{i}\left(\lambda_{i} u\right)\right)\left(\partial_{x_{j}} \sum \lambda_{i}^{2}\right)}{\left(\sum \lambda_{i}^{2}\right)^{2}}\right|^{p} d x\right)^{1 / p} \\
& \leq\left(\sum_{j} \int_{U^{*}}\left|\frac{\left(\sum \lambda_{i} E^{i}\left(\lambda_{i} u\right)\right)\left(2 \sum\left(\partial_{x_{j}} \lambda_{i}\right) \lambda_{i}\right)}{\left(\sum \lambda_{i}^{2}\right)^{2}}\right|^{p} d x\right)^{1 / p} \\
& \leq\left(\sum_{j} \int_{U^{*}}\left|\frac{\left(\sum \lambda_{i} E^{i}\left(\lambda_{i} u\right)\right)\left(2 b_{\varepsilon} N^{1 / 2}\left(\sum \lambda_{i}^{2}\right)^{\frac{1}{2}}\right)}{\left(\sum \lambda_{i}^{2}\right)^{2}}\right|^{p} d x\right)^{1 / p} \\
& \quad \leq 2 b_{\varepsilon} N^{1 / 2}\left(\sum_{j} \int_{U^{*}}\left|\sum \lambda_{i} E^{i}\left(\lambda_{i} u\right)\right|^{p} d x\right)^{1 / p} \\
& \leq 2 b_{\varepsilon} N^{3 / 2-1 / p} n^{1 / p}\left(\sum \int_{U_{i}}\left|E^{i}\left(\lambda_{i} u\right)\right|^{p} d x\right)^{1 / p} \\
& \leq 2 b_{\varepsilon} N^{3 / 2-1 / p} A n^{1 / p}\left(\sum \int_{\Omega}\left|\lambda_{i} u\right|^{p} d x\right)^{1 / p} \\
& \leq 2 b_{\varepsilon} N^{3 / 2} A n^{1 / p}\left(\int_{\Omega}|u|^{p} d x\right)^{1 / p} \cdot
\end{aligned}
$$


From these evaluations we have

$$
\begin{aligned}
\|\nabla(E u)\|_{L^{p}\left(\mathbb{R}^{n}\right)} & b_{+} N A n^{1 / p}\left(\int_{\Omega}|u|^{p} d x\right)^{1 / p}+N b_{\varepsilon} A n^{1 / p}\left(\int_{\Omega}|u|^{p} d x\right)^{1 / p} \\
& +N A^{\prime}\left\{b_{\varepsilon} n^{1 / p}\left(\int_{\Omega}|u|^{p} d x\right)^{1 / p}+\left(\int_{\Omega} \sum_{j}\left|\partial_{x_{j}} u\right|^{p} d x\right)^{1 / p}\right\} \\
& +2 N^{3 / 2} b_{\varepsilon} A n^{1 / p}\left(\int_{\Omega}|u|^{p} d x\right)^{1 / p}+b_{-} n^{1 / p}\left(\int_{\Omega}|u|^{p} d x\right)^{1 / p} \\
& +\left(\int_{\Omega} \sum_{j}\left|\partial_{x_{j}} u\right|^{p} d x\right)^{1 / p} \\
= & \left(N A^{\prime}+1\right)\left(\int_{\Omega} \sum_{j}\left|\partial_{x_{j}} u\right|^{p} d x\right)^{1 / p} \\
& +\left(b_{+} N A+b_{\varepsilon} N A+2 b_{\varepsilon} N^{3 / 2} A+b_{\varepsilon} N A^{\prime}+b_{-}\right) n^{1 / p}\left(\int_{\Omega}|u|^{p} d x\right)^{1 / p} \\
= & \left(N A^{\prime}+1\right)\|\nabla u\|_{L^{p}(\Omega)}+b_{\varepsilon}\left(4 N A+2 N^{3 / 2} A+N A^{\prime}+3\right) n^{1 / p}\|u\|_{L^{p}(\Omega) .}
\end{aligned}
$$

Hence, inequality (22) holds for

$$
A_{p}(\Omega)= \begin{cases}N A^{\prime}+1, & R \leq \gamma, \\ b_{\varepsilon}\left(4 N A+2 N^{3 / 2} A+N A^{\prime}+3\right) n^{1 / p} / \gamma, & R>\gamma,\end{cases}
$$

where $R:=b_{\varepsilon}\left(4 N A+2 N^{3 / 2} A+N A^{\prime}+3\right) n^{1 / p} /\left(N A^{\prime}+1\right)$.

The operator norm derived by Theorem 4.2 leads to bounds for the embedding constant as in the following corollary.

Corollary 4.3 For given $n \in\{2,3, \ldots\}$ and $p \in(n /(n-1), \infty)$, let $T_{p}$ be a constant satisfying $\|u\|_{L^{p}\left(\mathbb{R}^{n}\right)} \leq T_{p}\|\nabla u\|_{L^{q}\left(\mathbb{R}^{n}\right)}$ for all $u \in W^{1, q}\left(\mathbb{R}^{n}\right)$, where $q=n p /(n+p)$. Moreover, let $\Omega \subset \mathbb{R}^{n}$ be a domain with minimally smooth boundary. Then,

$$
\|u\|_{L^{p}(\Omega)} \leq C_{p}(\Omega)\|u\|_{W^{1, q}(\Omega)}, \quad \forall u \in W^{1, q}(\Omega)
$$

with

$$
C_{p}(\Omega)=2^{\frac{q-1}{q}} T_{p} A_{q}(\Omega)
$$

Here, $\|\cdot\|_{W^{1, q}(\Omega)}$ denotes the $\sigma$-weighted $W^{1, q}$ norm (2) for given $\sigma>0$, and $A_{q}(\Omega)$ is the upper bound of the operator norm derived by Theorem 4.2 with $\gamma=\sigma^{1 / q}$.

Proof We have

$$
\begin{aligned}
\|u\|_{L^{p}(\Omega)} & \leq\|E u\|_{L^{p}\left(\mathbb{R}^{n)}\right.} \\
& \leq T_{p}\|\nabla E u\|_{L^{q}\left(\mathbb{R}^{n}\right)}
\end{aligned}
$$




$$
\begin{aligned}
& \leq T_{p} A_{q}(\Omega)\left(\|\nabla u\|_{L^{q}(\Omega)}+\sigma^{1 / q}\|u\|_{L^{q}(\Omega)}\right) \\
& \leq 2^{\frac{q-1}{q}} T_{p} A_{q}(\Omega)\|u\|_{W^{1, q}(\Omega)}
\end{aligned}
$$

for all $u \in W^{1, q}(\Omega)$.

Remark 4.4 The value $A_{p}(\Omega)$ derived by Theorem 4.2 monotonically decreases with $\xi \in(0,1)$. Moreover, $\left.A_{p}(\Omega) \rightarrow A_{p}(\Omega)\right|_{\xi=0}(\xi \downarrow 0)$. Therefore, $\left.A_{p}(\Omega)\right|_{\xi=0}+\delta$ and $\left.C_{p}(\Omega)\right|_{\xi=0}+\delta$ for any positive number $\delta$ become an upper bound of the norm of the extension operator $E$ (defined by (12)) and an upper bound of the embedding constant (satisfying (1)), respectively, whereas the range of $\xi$ is $(0,1)$.

Remark 4.5 The constant $C_{p}^{\prime}(\Omega)$ such that $\|u\|_{L^{p}(\Omega)} \leq C_{p}^{\prime}(\Omega)\|u\|_{H^{1}(\Omega)}$ for all $u \in H^{1}(\Omega)$ is also important, especially for verified numerical computation and computer-assisted proof for PDEs summarized in, e.g., [9-11,13]. We can obtain a formula that gives a concrete value of $C_{p}^{\prime}(\Omega)$ with additional assumptions for $\Omega$ and $p$ (see Corollary D.1).

\section{Examples}

In this section, we present some examples of estimating the embedding constant $C_{p}(\Omega)$ defined in (1) using Theorem 4.2 and Corollary 4.3. Throughout this section, we set $\rho$ as the mollifier defined in (4) and $\sigma=1$.

\subsection{Calculation of constants}

The constants $A_{0}, A_{1}, P$, and $b_{\varepsilon}$ in Lemma 4.1 and Theorem 4.2 were numerically calculated. All computations were carried out on a computer with an Intel Core i7 $860 \mathrm{CPU}$ (2.80 GHz), 16.0 GB RAM, Windows 7, and MATLAB 2012b. Since all the rounding errors were strictly estimated using INTLAB version 6 [28], a toolbox for verified numerical computations, the accuracy of all results is mathematically guaranteed.

The constants $A_{0}$ and $A_{1}$ can be respectively computed as

$$
A_{0}=\sup \left\{\left|t^{2} \psi(t)\right|: t \geq 1\right\} \quad \text { and } \quad A_{1}=\sup \left\{\left|t^{3} \psi(t)\right|: t \geq 1\right\}
$$

with the function $\psi: \mathbb{R} \rightarrow \mathbb{R}$ constructing the extension operator (10), which satisfies property (11). For example, the function

$$
\psi(t):=\frac{e}{\pi t} \operatorname{Im}\left(e^{-\omega(t-1)^{\frac{1}{4}}}\right), \quad \omega=C_{\omega} e^{-\frac{i \pi}{4}}=\frac{C_{\omega}}{\sqrt{2}}(1-i)
$$

satisfies this property for any $C_{\omega}>0$; a simple proof can be seen in, e.g., [6, 12]. Table 1 lists values of $A_{0}$ and $A_{1}$ for some selections of $C_{\omega}$. We believe that $C_{\omega}=4.83$ is a 'good' (albeit not optimal) selection for obtaining small $A_{0}$ and $A_{1}$. Moreover, recall that $b_{\varepsilon}$ is a positive number satisfying

$$
b_{\varepsilon} \geq \int_{\mathbb{R}^{n}}\left|\partial_{x_{j}} \rho_{\frac{1}{4} \varepsilon}(x)\right| d x\left(=\frac{4}{\varepsilon} \int_{\mathbb{R}^{n}}\left|\partial_{x_{1}} \rho(x)\right| d x\right) .
$$

For the mollifier defined in (4), the bounds for the integration in (32) are independent of the index $j$. Furthermore, one of the concrete values of $P$ can be derived by (7) with the 
Table 1 Values of $A_{0}$ and $A_{1}$

\begin{tabular}{lll}
\hline $\boldsymbol{C}_{\boldsymbol{\omega}}$ & $\boldsymbol{A}_{\boldsymbol{0}} \boldsymbol{\epsilon}$ & $\boldsymbol{A}_{\mathbf{1}} \boldsymbol{\epsilon}$ \\
\hline 1 & {$[15.4893767258475,15.4893767258481]$} & {$[77833.36333368837,77833.3633336869]$} \\
2 & {$[2.65390726696554,2.653907266965600]$} & {$[828.073610866384,828.073610866409]$} \\
3 & {$[2.10591300732044,2.10591300732053]$} & {$[88.5738560955430,88.5738560955457]$} \\
4 & {$[5.63766113155191,5.63766113155202]$} & {$[24.6566936219851,24.6566936219859]$} \\
4.83 & {$[12.8860220862419,12.8860220862429]$} & {$[12.9832586501139,12.9832586501147]$} \\
5 & {$[15.2681658080940,15.2681658080947]$} & {$[15.30722743369907,15.3072274336919]$} \\
\hline
\end{tabular}

condition $|\alpha|=1$, i.e., it can be computed as

$$
P=\int_{\mathbb{R}^{n}}\left\{(n-1) \rho_{*}(|x|)+|x| \rho_{*}^{\prime}(|x|)\right\}(1-|x|)^{-1} d x .
$$

Using verified numerical computation, we derived the following estimation results:

$$
\int_{\mathbb{R}^{2}}\left|\partial_{x_{1}} \rho(x)\right| d x \in[1.86412,1.92770] \quad \text { and } \quad P \in[7.45592,7.50131]
$$

for the case of $n=2$.

\subsection{Examples of estimating the embedding constant}

Here, we present estimation results for the following two concrete domains:

Example A Let $\Omega \subset \mathbb{R}^{2}$ be the domain as in Figure 1(a). We set $\left\{U_{i}\right\}_{i \in \mathbb{N}}$ as follows: we first defined the two sets among $U_{i}$ as in Figure $1(\mathrm{~b})$; then, $U_{i}(i=1,2, \ldots, 8)$ were obtained by symmetry reflections; and finally, we defined the other $U_{i}(i=9,10,11, \ldots)$ as empty sets. In this case, we chose $M=1, N=2$, and $\varepsilon=0.25$. We can find in Figure 1(c) that these constants satisfy the required conditions mentioned in Theorem 4.2.

Figure 2(a) shows the relationship between $\tau$ and $A_{q}(\Omega)$ in the cases of $p=4,6$, and 8; recall that $q=2 p /(2+p)$. We can observe that $A_{q}(\Omega)$ first decreases as $\tau$ increases. Then, it reaches a minimum point; thereafter, it monotonically increases with $\tau$. The relationship between $p$ and the value of $\tau$ that minimizes $A_{q}(\Omega)$ can be seen in Figure 2(b). For example, in the cases of $p=4,6$, and 8 , each $A_{q}(\Omega)$ is minimized at the points $\tau \approx 8.12,5.83$, and 5.06 , respectively.

Figure 2(c) shows the relationship between $p$ and $C_{p}(\Omega)$; we chose $\tau$ such that $A_{q}(\Omega)$ (and $\left.C_{p}(\Omega)\right)$ are as small as possible. Recall that all the results in Figure 2 are mathematically guaranteed with verified numerical computation.

Example B Let $\Omega \subset \mathbb{R}^{2}$ be the domain as in Figure 3(a), the boundary of which is composed of five semicircles and a straight line. We set $\left\{U_{i}\right\}_{i \in \mathbb{N}}$ as follows: we first set $U_{i}$ $(i=1,2, \ldots, 6)$ as in Figures 3(b), (c), (d); then, we obtained the other $U_{i} \mathrm{~s}(i=7,8, \ldots, 10)$ by symmetrical reflection; and finally, the other $U_{i}(i=11,12, \ldots)$ were defined as empty sets. In this case, we chose $M=1, N=2$, and $\varepsilon=2 \sin (\pi / 8) /\{\sin (\pi / 8)+1\}$. The selection of $\varepsilon$ depends on the smallest semicircle that constitutes the boundary of $\Omega$. We can find in Figure 4 that $\varepsilon=2 \sin (\pi / 8) /\{\sin (\pi / 8)+1\}$ satisfies the required condition in Theorem 4.2. The graphs of $A_{q}(\Omega), \tau$ that minimizes $A_{q}(\Omega)$, and $C_{p}(\Omega)$ are also shown in Figure 5 . 


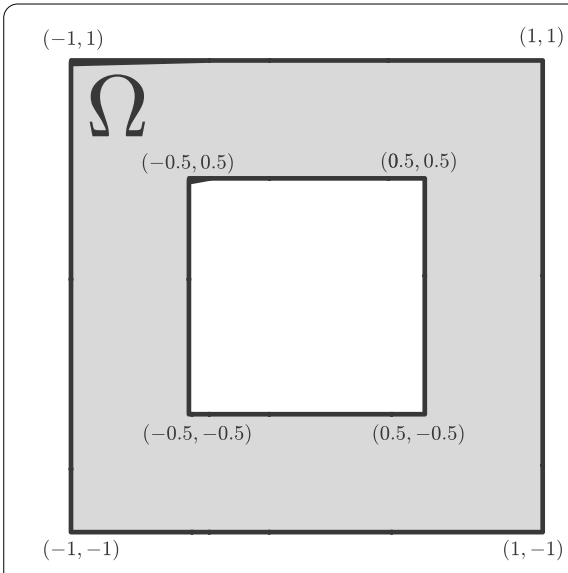

(a)

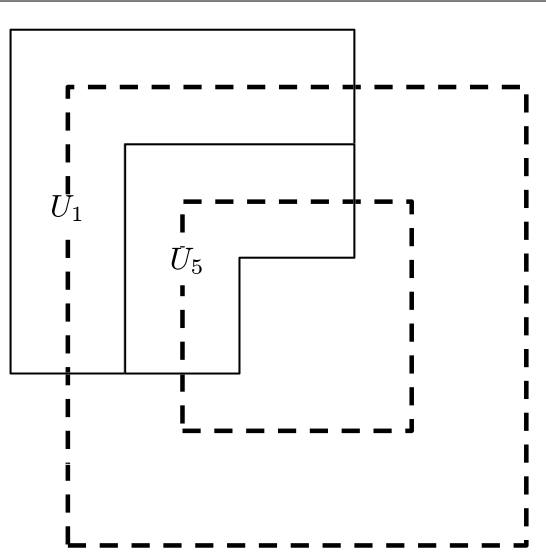

(b)

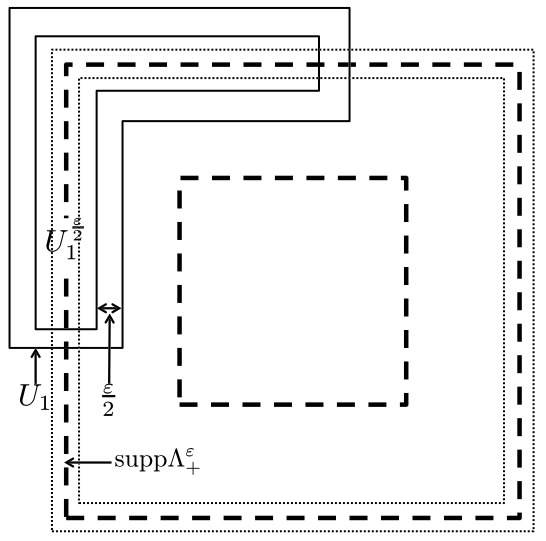

(c)

Figure 1 Figures in Example A. (a): Domain $\Omega$. (b) and (c): open sets $U_{i}(i=1,2, \ldots, 8)$.

\section{Conclusion}

We proposed a method for estimating the operator norm $A_{q}(\Omega)$ (defined in (3)) of the extension operator constructed by Stein [12]. The concrete bounds for the operator norm lead to estimation of the embedding constant $C_{p}(\Omega)$ from $W^{1, q}(\Omega)$ to $L^{p}(\Omega)$, as defined in (1). Here, $\Omega$ is only assumed to be a domain with minimally smooth boundary.

In addition, we presented some estimation results of the embedding constants. All the estimation results are mathematically guaranteed with verified numerical computation, whereas the derived constants may not be sharp because of some overestimations.

\section{Appendix 1: Relation between $p$-norms}

The following lemma is required for proving Corollary B.2 and Corollary D.1.

Lemma A.1 For $0<p<q$ and $x \in \mathbb{R}^{n}(n \in \mathbb{N})$, we have

$$
|x|_{q} \leq|x|_{p} \leq n^{\frac{1}{p}-\frac{1}{q}}|x|_{q}
$$

where both inequalities are optimal. 


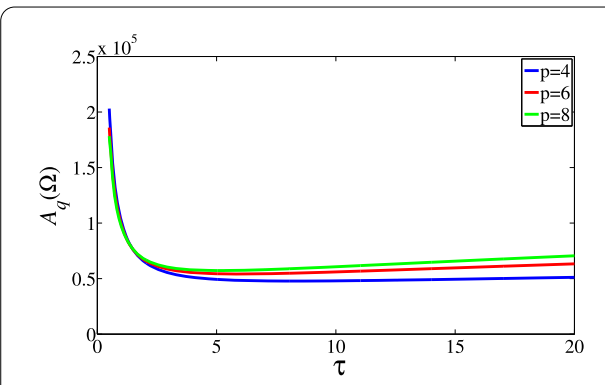

(a)

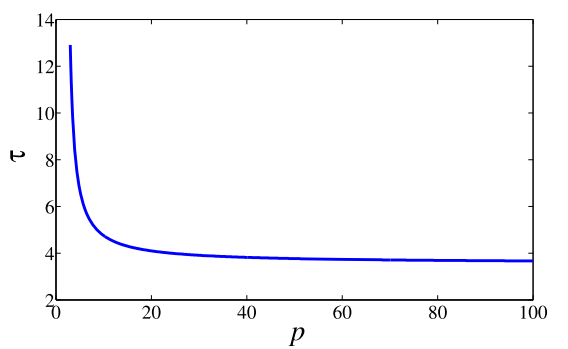

(b)

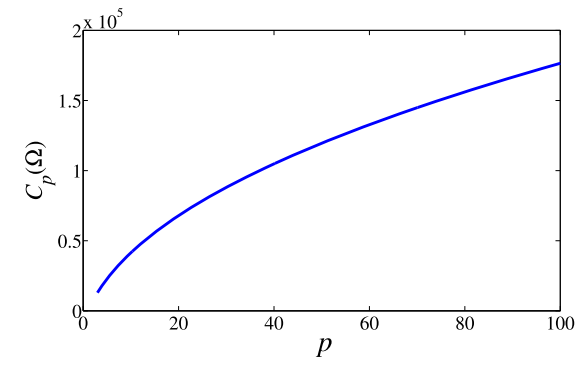

(c)

Figure 2 The relationship between (a) $\tau$ and $A_{q}(\Omega)$ with $p=4,6$, and 8 , (b) $p$ and $\tau$ that minimizes $A_{q}(\Omega)$, and (c) $p$ and $C_{p}(\Omega)$.

Proof We first prove the left inequality in (33). This is clear when $x=0$. Otherwise, since $\left|x_{i}\right| /|x|_{q} \leq 1$ for $i=1,2, \ldots, n$, we have

$$
\sum_{i=1}^{n}\left(\frac{\left|x_{i}\right|}{|x|_{q}}\right)^{p} \geq \sum_{i=1}^{n}\left(\frac{\left|x_{i}\right|}{|x|_{q}}\right)^{q}=1
$$

and therefore, $|x|_{p} \geq|x|_{q}$. The equality is attained, e.g., when $x_{1}=1$ and $x_{i}=0(i=$ $2,3, \ldots, n)$.

We then prove the right inequality in (33). Holder's inequality ensures that

$$
|x|_{p}^{p} \leq\left(\sum_{i=1}^{n}\left(\left|x_{i}\right|^{p}\right)^{\frac{q}{p}}\right)^{\frac{p}{q}}\left(\sum_{i=1}^{n} 1^{\frac{q}{q-p}}\right)^{\frac{q-p}{q}}=n^{1-\frac{p}{q}}\left(\sum_{i=1}^{n}\left|x_{i}\right|^{q}\right)^{\frac{p}{q}} .
$$

Raising both sides of this inequality to the power $1 / p$, we have $|x|_{p} \leq n^{\frac{1}{p}-\frac{1}{q}}|x|_{q}$. The equality is attained, e.g., when $x_{i}=1(i=1,2, \ldots, n)$.

\section{Appendix 2: The best constant in the classical Sobolev inequality}

The following theorem gives the best constant in the classical Sobolev inequality.

Theorem B.1 (Aubin [1] and Talenti [14]) Let $u$ be any function in $H^{1}\left(\mathbb{R}^{n}\right)(n=2,3, \ldots)$. Moreover, let $q$ be any real number such that $1<q<n$, and set $p=n q /(n-q)$. Then,

$$
\left(\int_{\mathbb{R}^{n}}|u(x)|^{p} d x\right)^{\frac{1}{p}} \leq T_{p}^{\prime}\left(\int_{\mathbb{R}^{n}}|\nabla u(x)|_{2}^{q} d x\right)^{\frac{1}{q}}
$$




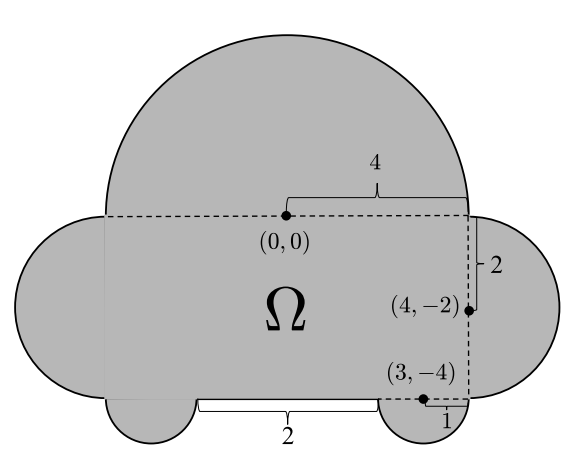

(a)

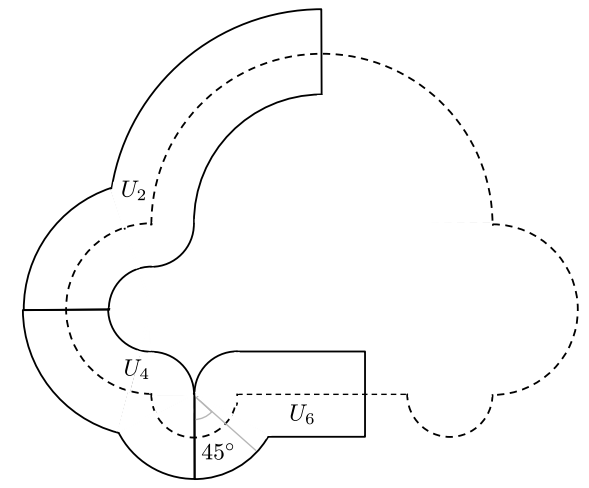

(c)

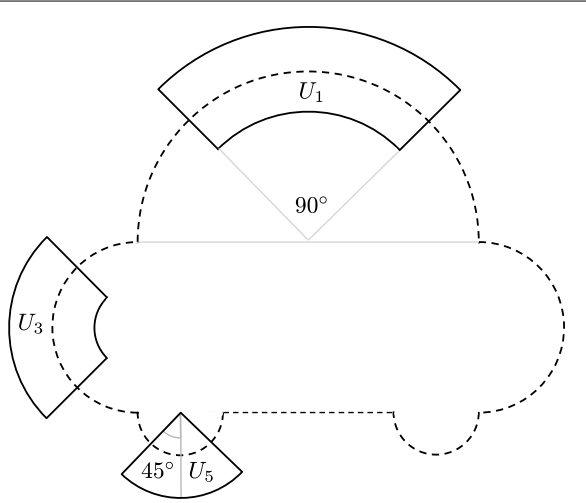

(b)

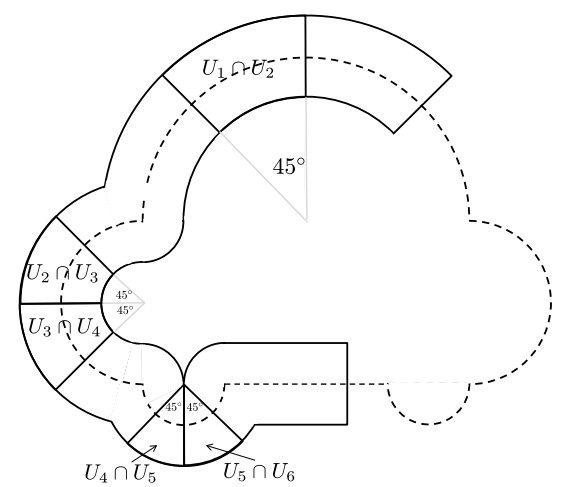

(d)

Figure 3 Figures in Example B. (a): Domain $\Omega$. (b)-(d): $U_{i}(i=1,2, \ldots, 6)$ are shown; the other $U_{i}(i=7,8, \ldots, 10)$ can be obtained by symmetrical reflection.

Figure 4 How to determine $\varepsilon$.

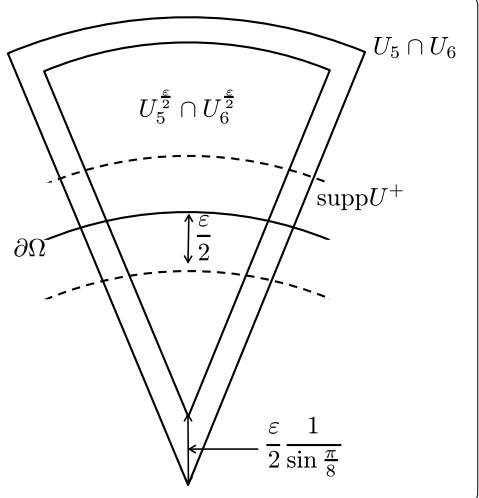

with

$$
T_{p}^{\prime}=\pi^{-\frac{1}{2}} n^{-\frac{1}{q}}\left(\frac{q-1}{n-q}\right)^{1-\frac{1}{q}}\left\{\frac{\Gamma\left(1+\frac{n}{2}\right) \Gamma(n)}{\Gamma\left(\frac{n}{q}\right) \Gamma\left(1+n-\frac{n}{q}\right)}\right\}^{\frac{1}{n}},
$$

where $\Gamma$ is the gamma function.

From Lemma A.1 and Theorem B.1 the following corollary immediately follows. 


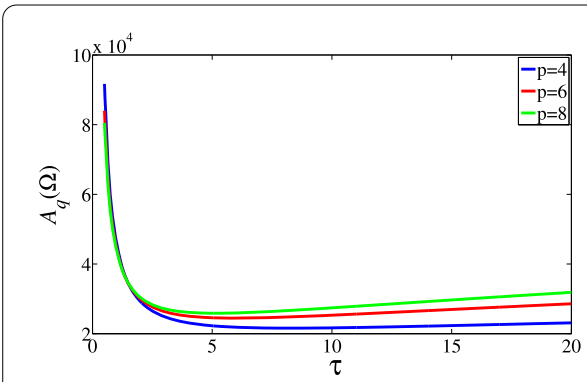

(a)

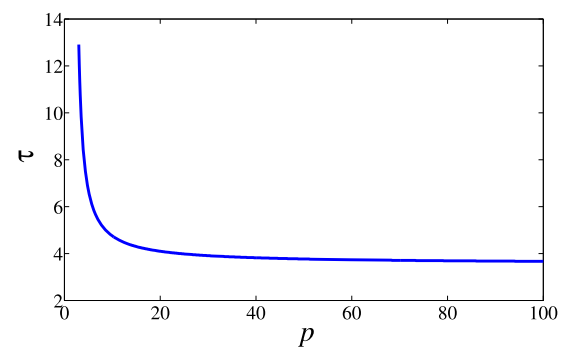

(b)

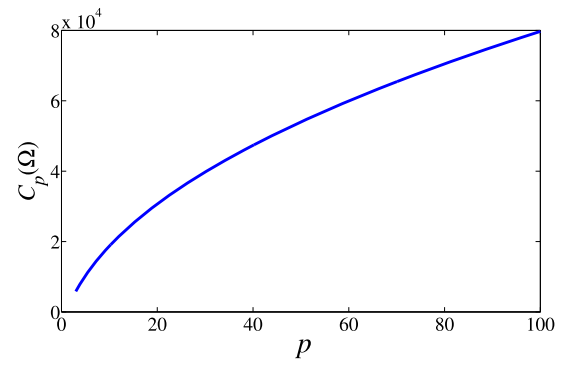

(c)

Figure 5 The same as Figure 2, but for the case of the domain $\Omega$ in Figure 3(a).

Corollary B.2 Let $u$ be any function in $H^{1}\left(\mathbb{R}^{n}\right)(n=2,3, \ldots)$. Moreover, let $q$ be any real number such that $1<q<n$, and set $p=n q /(n-q)$. Then,

$$
\|u\|_{L^{p}\left(\mathbb{R}^{n}\right)} \leq T_{p}\|\nabla u\|_{L^{q}\left(\mathbb{R}^{n}\right)}
$$

with $T_{p}=M_{2, q} T_{p}^{\prime}$, where $T_{p}^{\prime}$ is defined by (34), and

$$
M_{2, q}:= \begin{cases}1, & 1<q<2 \\ n^{\frac{1}{2}-\frac{1}{q}}, & 2 \leq q<n .\end{cases}
$$

Proof Since Lemma A.1 ensures that

$$
\left(\int_{\mathbb{R}^{n}}|\nabla u(x)|_{2}^{q} d x\right)^{\frac{1}{q}} \leq M_{2, q}\left(\int_{\mathbb{R}^{n}}|\nabla u(x)|_{q}^{q} d x\right)^{\frac{1}{q}}=M_{2, q}\|\nabla u\|_{L^{q}\left(\mathbb{R}^{n}\right)},
$$

this corollary holds.

\section{Appendix 3: Lemmas for proving Lemma 4.1 and Theorem 4.2}

The following two lemmas are required to prove Lemma 4.1 and Theorem 4.2.

Lemma C.1 (Hardy et al. [29]) Let $p \in \mathbb{N}$ and $r>0$. Suppose that a function $f: \mathbb{R} \rightarrow \mathbb{R}$ satisfies $f(x) \geq 0, \forall x \in \mathbb{R}$. Then, it follows that

$$
\left(\int_{0}^{\infty}\left(\int_{0}^{x} f(y) d y\right)^{p} x^{-r-1} d x\right)^{1 / p} \leq \frac{p}{r}\left(\int_{0}^{\infty}(y f(y))^{p} y^{-r-1} d y\right)^{1 / p}
$$


and

$$
\left(\int_{0}^{\infty}\left(\int_{x}^{\infty} f(y) d y\right)^{p} x^{r-1} d x\right)^{1 / p} \leq \frac{p}{r}\left(\int_{0}^{\infty}(y f(y))^{p} y^{r-1} d y\right)^{1 / p} .
$$

Lemma C.2 Let $S \subseteq \mathbb{R}^{n}$ and $p \in[1, \infty)$. Moreover, let $\left\{a_{i}(x)\right\}_{i \in \mathbb{N}} \subset L^{p}(S)$ be such that at most $N$ of $a_{i}(x)$ are not zero for each $x$. Then, we have

$$
\left(\int_{S}\left|\sum_{i \in \mathbb{N}} a_{i}(x)\right|^{p} d x\right)^{\frac{1}{p}} \leq N^{1-\frac{1}{p}}\left(\sum_{i \in \mathbb{N}} \int_{S}\left|a_{i}(x)\right|^{p} d x\right)^{\frac{1}{p}}
$$

Proof This lemma follows from the inequality

$$
\left|\sum_{i \in \mathbb{N}} a_{i}(x)\right|^{p} \leq N^{p-1} \sum_{i \in \mathbb{N}}\left|a_{i}(x)\right|^{p}
$$

which comes from Hölder's inequality.

\section{Appendix 4: The embedding constant from $H^{1}(\Omega)$ to $L^{p}(\Omega)$}

Corollary D.1, which comes from Theorem 4.2, gives a concrete estimation of the embedding constant from $H^{1}(\Omega)$ to $L^{p}(\Omega)$ under suitable assumptions for $\Omega$ and $p$.

Corollary D.1 For given $n \in\{2,3, \ldots\}$, let $p$ be a real number such that $p \in(n /(n-$ $1), 2 n /(n-2))$ if $n \geq 3$ and $p \in(n /(n-1), \infty)$ if $n=2$, and set $q=n p /(n+p)$. Moreover, let $\Omega \subset \mathbb{R}^{n}$ be a bounded domain with minimally smooth boundary. Then, for any $r \in[q, 2)$,

$$
\|u\|_{L^{p}(\Omega)} \leq C_{p}^{\prime}(\Omega)\|u\|_{H^{1}(\Omega)}, \quad \forall u \in H^{1}(\Omega)
$$

with

$$
C_{p}^{\prime}(\Omega)=2^{1 / 2}|\Omega|^{\frac{2-q}{2 q}} T_{r^{*}} M_{r, 2} A_{r}(\Omega)
$$

Here, $\|\cdot\|_{H^{1}(\Omega)}$ denotes the $\sigma$-weighted $W^{1,2}$ norm (2) for given $\sigma>0, r^{*}:=n r /(n-r), T_{r^{*}}$ is a constant satisfying $\|u\|_{L^{r^{*}}\left(\mathbb{R}^{n}\right)} \leq T_{r^{*}}\|\nabla u\|_{L^{r}\left(\mathbb{R}^{n}\right)}$ for all $u \in W^{1, r}\left(\mathbb{R}^{n}\right), M_{r, 2}:=n^{\frac{1}{r}-\frac{1}{2}}$, and $A_{r}(\Omega)$ is the upper bound for the operator norm derived by Theorem 4.2 with $\gamma=\sigma^{1 / 2}$.

Proof Since $q \in(1,2)$, a real number $r$ such that $r \in[q, 2)$ exists.

Let $u \in H^{1}(\Omega)$. Hölder's inequality gives

$$
\|u\|_{L^{p}(\Omega)}^{p} \leq\left(\int_{\Omega}|u(x)|^{p \cdot \frac{r^{*}}{p}}\right)^{\frac{p}{r^{*}}}\left(\int_{\Omega} 1^{\frac{r^{*}}{r^{*}-p}}\right)^{\frac{r^{*}-p}{r^{*}}}=|\Omega|^{\frac{r^{*}-p}{r^{*}}}\|u\|_{L^{r^{*}}(\Omega)}^{p},
$$

and we have

$$
\begin{aligned}
\|u\|_{L^{r^{*}}(\Omega)} & \leq\|E u\|_{L^{r *\left(\mathbb{R}^{n}\right)}} \leq T_{r^{*}}\|\nabla E u\|_{L^{r}\left(\mathbb{R}^{n}\right)} \\
& \leq T_{r^{*}} A_{r}(\Omega)\left(\|\nabla u\|_{L^{r}(\Omega)}+\sigma^{1 / 2}\|u\|_{L^{r}(\Omega)}\right) .
\end{aligned}
$$


Therefore, it follows that

$$
\|u\|_{L^{p}(\Omega)} \leq|\Omega|^{\frac{r^{*}-p}{p r^{*}}} T_{r^{*}} A_{r}(\Omega)\left(\|\nabla u\|_{L^{r}(\Omega)}+\sigma^{1 / 2}\|u\|_{L^{r}(\Omega)}\right) .
$$

Moreover, Hölder's inequality again gives

$$
\begin{aligned}
\|\nabla u\|_{L^{r}(\Omega)}^{r} & \leq\left(\int_{\Omega}|\nabla u(x)|_{r}^{r \cdot \frac{2}{r}} d x\right)^{\frac{r}{2}}\left(\int_{\Omega} 1^{\frac{2}{2-r}} d x\right)^{\frac{2-r}{2}} \\
& =|\Omega|^{\frac{2-r}{2}}\left(\int_{\Omega}|\nabla u(x)|_{r}^{2} d x\right)^{\frac{r}{2}}
\end{aligned}
$$

where $|\Omega|$ is the measure of $\Omega$. Therefore, it follows from Lemma A.1 that

$$
\|\nabla u\|_{L^{r}(\Omega)} \leq|\Omega|^{\frac{2-r}{2 r}} M_{r, 2}\|\nabla u\|_{L^{2}(\Omega)}
$$

In the same manner, we have

$$
\|u\|_{L^{r}(\Omega)} \leq|\Omega|^{\frac{2-r}{2 r}}\|u\|_{L^{2}(\Omega)}
$$

Since $M_{r, 2} \geq 1$ and $\left(r^{*}-p\right) /\left(p r^{*}\right)+(2-r) /(2 r)=(2-q) /(2 q)$, it follows from (35), (36), and (37) that

$$
\begin{aligned}
\|u\|_{L^{p}(\Omega)} & \leq|\Omega|^{\frac{r^{*}-p}{p r^{*}}+\frac{2-r}{2 r}} T_{r^{*}} M_{r, 2} A_{r}(\Omega)\left(\|\nabla u\|_{L^{2}(\Omega)}+\sigma^{1 / 2}\|u\|_{L^{2}(\Omega)}\right) \\
& \leq 2^{1 / 2}|\Omega|^{\frac{2-q}{2 q}} T_{r^{*}} M_{r, 2} A_{r}(\Omega)\|u\|_{H^{1}(\Omega)} .
\end{aligned}
$$

\section{Competing interests}

The authors declare that they have no competing interests.

\section{Authors' contributions}

All the authors contributed equally. All authors read and approved the final manuscript.

\section{Author details}

${ }^{1}$ Graduate School of Fundamental Science and Engineering, Waseda University, 3-4-1 Okubo, Shinjuku-ku, Tokyo, 169-8555, Japan. '2Faculty of Science and Engineering, Waseda University, 3-4-1 Okubo, Shinjuku-ku, Tokyo, 169-8555, Japan. ${ }^{3}$ CREST/JST, Tokyo, Japan.

\section{Acknowledgements}

The authors express their sincere thanks to Professor M Kashiwagi (Waseda University, Tokyo, Japan) for his valuable comments. They also express their profound gratitude to an editor and two anonymous referees for their highly insightful comments and suggestions.

Received: 9 June 2015 Accepted: 24 November 2015 Published online: 09 December 2015

\section{References}

1. Aubin, T: Problèmes isopérimétriques et espaces de Sobolev. J. Differ. Geom. 11(4), 573-598 (1976)

2. Aubin, T: Nonlinear Analysis on Manifold. Monge-Ampère Equations. Springer, New York (1982)

3. Beckner, W: Sharp Sobolev inequalities on the sphere and the Moser-Trudinger inequality. Ann. Math. 138(1), 213-242 (1993)

4. Brezis, H, Nirenberg, L: Positive solutions of nonlinear elliptic equations involving critical Sobolev exponents. Commun. Pure Appl. Math. 36(4), 437-477 (1983)

5. Calderón, AP: Lebesgue spaces of differentiable functions and distributions. In: Proc. Sympos. Pure Math., vol. 4 , pp. 33-49 (1961)

6. Edmunds, DE, Evans, W: Spectral Theory and Differential Operators. Oxford University Press, New York (1987)

7. Hestenes, MR: Extension of the range of a differentiable function. Duke Math. J. 8(1), 183-192 (1941)

8. Lieb, EH: Sharp constants in the Hardy-Littlewood-Sobolev and related inequalities. Ann. Math. 118, 349-374 (1983) 
9. Nakao, MT: Numerical verification methods for solutions of ordinary and partial differential equations. Numer. Funct. Anal. Optim. 22(3-4), 321-356 (2001)

10. Plum, M: Computer-assisted enclosure methods for elliptic differential equations. Linear Algebra Appl. 324(1), 147-187 (2001)

11. Plum, M: Existence and multiplicity proofs for semilinear elliptic boundary value problems by computer assistance. Jahresber. Dtsch. Math.-Ver. 110(1), 19-54 (2008)

12. Stein, E: Singular Integrals and Differentiability Properties of Functions. Princeton University Press, Princeton (1970)

13. Takayasu, A, Liu, X, Oishi, S: Verified computations to semilinear elliptic boundary value problems on arbitrary polygonal domains. Nonlinear Theory Appl., IEICE 4(1), 34-61 (2013)

14. Talenti, G: Best constant in Sobolev inequality. Ann. Mat. Pura Appl. 110(1), 353-372 (1976)

15. Watanabe, K, Kametaka, Y, Nagai, A, Yamagishi, H, Takemura, K: Symmetrization of functions and the best constant of 1-dim Sobolev inequality. J. Inequal. Appl. 2009, 874631 (2009)

16. Whitney, H: Analytic extensions of differentiable functions defined in closed sets. Trans. Am. Math. Soc. 36(1), 63-89 (1934)

17. Cai, S, Nagatou, K, Watanabe, Y: A numerical verification method for a system of FitzHugh-Nagumo type. Numer. Funct. Anal. Optim. 33(10), 1195-1220 (2012)

18. Adams, RA, Fournier, JJ: Sobolev Spaces, 2nd edn. Pure and Applied Mathematics. Academic Press, New York (2003)

19. Brezis, H: Functional Analysis, Sobolev Spaces and Partial Differential Equations. Springer, New York (2011)

20. Rogers, LG: Degree-independent Sobolev extension on locally uniform domains. J. Funct. Anal. 235(2), 619-665 (2006)

21. Jones, PW: Quasiconformal mappings and extendability of functions in Sobolev spaces. Acta Math. 147(1), 71-88 (1981)

22. Azzam, J, Schul, R: Hard Sard: quantitative implicit function and extension theorems for Lipschitz maps. Geom. Funct. Anal. 22(5), 1062-1123 (2012)

23. Fefferman, C: Whitney's extension problem for $C^{m}$. Ann. Math. 164(1), 313-359 (2006)

24. Hiptmair, $R, L i, J, Z o u, J:$ Universal extension for Sobolev spaces of differential forms and applications. J. Funct. Anal. 263(2), 364-382 (2012)

25. Pechstein, C: Shape-explicit constants for some boundary integral operators. Appl. Anal. 92(5), 949-974 (2013)

26. Fraenkel, L: On regularized distance and related functions. Proc. R. Soc. Edinb., Sect. A, Math. 83(1-2), 115-122 (1979)

27. Grisvard, P: Elliptic Problems in Nonsmooth Domains. Classics Appl. Math. SIAM, Philadelphia (2011)

28. Rump, S: INTLAB - INTerval LABoratory. In: Csendes, T (ed.) Developments in Reliable Computing, pp. 77-104. Kluwer Academic, Dordrecht (1999) http://www.ti3.tuhh.de/rump/

29. Hardy, GH, Littlewood, JE, Pólya, G: Inequalities. Cambridge University Press, Cambridge (1952)

\section{Submit your manuscript to a SpringerOpen ${ }^{\ominus}$ journal and benefit from:}

- Convenient online submission

Rigorous peer review

- Immediate publication on acceptance

- Open access: articles freely available online

- High visibility within the field

- Retaining the copyright to your article 